\title{
ONC201: a new treatment option being tested clinically for recurrent glioblastoma
}

\author{
Marie D. Ralff ${ }^{1,2}$, Amriti R. Lulla ${ }^{2,3}$, Jessica Wagner ${ }^{2,4}$, Wafik S. El-Deiry ${ }^{2}$ \\ ${ }^{1} \mathrm{MD} / \mathrm{PhD}$ Program, Lewis Katz School of Medicine, Temple University, Philadelphia, PA, USA; ${ }^{2}$ Laboratory of Translational Oncology and \\ Experimental Cancer Therapeutics, Department of Medical Oncology and Molecular Therapeutics Program, Fox Chase Cancer Center, \\ Philadelphia, PA, USA; ${ }^{3}$ PhD Program, Penn State Hershey Cancer Institute, Penn State College of Medicine, Hershey, PA, USA; ${ }^{4}$ PhD Program, \\ Lewis Katz School of Medicine, Temple University, Philadelphia, PA, USA \\ Correspondence to: Wafik S. El-Deiry. Laboratory of Translational Oncology and Experimental Cancer Therapeutics, Department of Medical \\ Oncology and Molecular Therapeutics Program, Fox Chase Cancer Center, 333 Cottman Avenue, Room P2035, Philadelphia, PA 19111, USA. \\ Email: wafik.eldeiry@fccc.edu. \\ Comment on: Arrillaga-Romany I, Chi AS, Allen JE, et al. A phase 2 study of the first imipridone ONC201, a selective DRD2 antagonist for oncology, \\ administered every three weeks in recurrent glioblastoma. Oncotarget 2017;8:79298-304.
}

\begin{abstract}
Glioblastoma is an aggressive central nervous system tumor with a 5-year-survival rate of less than $10 \%$. Patients diagnosed with the disease are treated with surgery, radiation and temozolomide chemotherapy. Despite survival benefits, patients eventually relapse. There is a need for new treatments with improved efficacy. Imipridone ONC201 is a small molecule originally identified as a TNF-related apoptosis inducing ligand (TRAIL)-inducing compound. ONC201 has the unique ability to induce expression of both pro-death ligand TRAIL and its receptor DR5 through engagement of the cellular integrated stress response (ISR) pathway. Arrillaga-Romany et al. report early results from futility analysis of a phase II clinical trial of ONC201 in 17 patients with recurrent or refractory glioblastoma conducted at the Massachusetts General Hospital Cancer Center. The results are promising, as ONC201 shows preliminary signs of efficacy. Further testing of ONC201 in an expansion cohort of patients with glioblastoma is ongoing.
\end{abstract}

Keywords: ONC201; glioblastoma; clinical trial

Submitted Sep 05, 2017. Accepted for publication Sep 25, 2017.

doi: $10.21037 /$ tcr.2017.10.03

View this article at: http://dx.doi.org/10.21037/tcr.2017.10.03

\section{Background}

Glioblastoma is a high-grade tumor of the central nervous system (CNS). Prognosis for patients diagnosed with this deadly cancer is poor, with a 5 -year-survival rate of $9.8 \%$ (1). Affected patients often present with generalized CNS symptoms that worsen over time including headaches and seizures. Contrast-enhanced magnetic resonance imaging (MRI) is performed to confirm the presence of an abnormal mass, but a proper diagnosis requires analysis of a tumor sample by a pathologist. This sample can be obtained through biopsy or at the time of surgical resection. Pathologists grade glial derived tumors according to the WHO classification system (2), with glioblastoma being the highest grade (WHO grade IV). Glioblastomas can be primary, appearing in the absence of a precursor lesion, or secondary, progressing from lower grade glial tumors.

All eligible patients will have their glioblastoma surgically resected with the goal of removing as much of the malignancy as possible while preserving neurological function. Observational studies have shown that extent of resection is a prognostic factor, and that a more complete resection confers an improved prognosis (3). Despite the clear benefits of surgery, glioblastoma is an infiltrative tumor and at the time of diagnosis cancer cells have often spread from the primary lesion and into the adjacent brain tissue. Adjuvant radiation followed by temozolomide chemotherapy is given as the standard of care with the goal of eradicating this residual disease. Addition of radiation to surgical resection prolongs overall survival (OS) in 
glioblastoma patients (4). Patients typically receive involved field radiation therapy (IFRT). Unlike whole brain radiation therapy (WBRT), IFRT irradiates only the tumor site and a 2-cm margin of surrounding brain tissue. The addition of alkylating agent temozolomide to radiation further prolongs survival $(1,5)$. Molecular alterations with prognostic and predictive value have been identified in glioblastoma tumors. Methylation of the $\mathrm{O}^{6}$-methylguanine-DNA methyltransferase (MGMT) promoter correlates with an overall better prognosis (6). The MGMT gene encodes a DNA-repair protein that removes alkyl groups from the $\mathrm{O}^{6}$ position of guanine. Epigenetic silencing of this gene leads to increased DNA damage following treatment with alkylating chemotherapy (7) such as temozolomide.

Despite the survival benefits associated with adjuvant use of radiation and temozolomide, almost all patients will eventually relapse. Single cell sequencing has revealed extensive intratumoral heterogeneity within individual glioblastoma tumors (8). Glioblastoma tumors contain a population of cells capable of self-renewal and known as cancer stem cells (CSCs). There is data suggesting that glioblastoma CSCs are responsible for tumor relapse (9), and they are highly resistant to both chemotherapy and radiation. Relapsed glioblastoma patients can be treated with reoperation and re-irradiation, but these interventions have significant risks and are ultimately palliative. As systemic therapy for relapsed glioblastoma, patients currently have the option of receiving bevacizumab, nitrosoureas, or a rechallenge with temozolomide. Bevacizumab, a humanized monoclonal antibody targeting VEGF-A, is associated with a wide variety of toxicities and has not demonstrated an ability to improve OS in this setting $(10,11)$. Nitrosourea based chemotherapy and temozolomide rechallenge are also associated with significant toxicities and do not dramatically extend survival. Clearly there is a significant shortage of therapies that are both nontoxic and efficacious for patients with relapsed glioblastoma.

\section{Preclinical activity of ONC201 in glioblastoma}

ONC201 is the first member of the imipridone class of small molecules $(12,13)$. The compound is currently being tested in phase II clinical trials of patients with hematological malignancies and solid tumors $(14,15)$. ONC201 was discovered in a phenotypic screen for compounds that could transcriptionally induce the TNF-related apoptosis inducing ligand (TRAIL) gene in colon cancer cells, independent of p53 status $(16,17)$. ONC201 has since then demonstrated the ability to potently and selectively induce growth arrest and apoptosis in many different tumor types, with limited or no toxicity to normal cells $(16,18-20)$. The integrated stress response (ISR) is important in the pro-apoptotic effects of ONC201, with transcription factors ATF4 and CHOP involved in upregulation of TRAIL receptor DR5 $(21,22)$. ONC201 has also demonstrated single agent efficacy in eliminating CSCs in colorectal, glioblastoma and prostate cancer models $(23,24)$. The binding target of ONC201 was unknown until recent computational studies predicted antagonism of dopamine receptors. In vitro binding studies have confirmed the compound to be direct competitive antagonist of D2-like receptors dopamine receptors DRD2 and DRD3 with a binding affinity $\left(K_{i}\right)$ of $3 \mu M(25)$.

The earliest preclinical studies conducted with ONC201 showed signs of efficacy against glioblastomas (Figure 1). ONC201 upregulates TRAIL and induces apoptosis in several glioblastoma cell lines, including those resistant to standard of care therapy temozolomide. In addition, ONC201 shows potent cytotoxicity in bevacizumab, temozolomide, and radiation resistant glioblastoma patient samples. More recently, the compound was shown to inhibit the growth of glioblastoma CSC cells in $3 \mathrm{D}$ neurosphere cultures, established from freshly isolated human glioblastoma tumors. Most importantly, studies in glioblastoma mouse models have shown that ONC201 crosses the blood brain barrier and inhibits tumor growth (16). The first-in-human trial of ONC201 determined the recommended phase II dose (RP2D) to be $625 \mathrm{mg}$, orally administered every three weeks. This dose was well tolerated and biologically active. Within hours of ONC201 treatment, levels of caspase-cleaved keratin 18 and prolactin (a marker of DRD2 antagonism) were increased in patient serum. Several treated patients showed radiographic regression of metastatic disease as well as prolonged stable disease (15). Together, these preclinical and clinical data indicate that ONC201 is an exciting and novel candidate therapy that merits clinical evaluation in patients with glioblastoma.

\section{Clinical activity of ONC201 in glioblastoma}

In a recently published article (26), Arrillaga-Romany et al. describe the results of a phase II clinical trial of imipridone compound ONC201 in patients with recurrent bevacizumab-naïve glioblastoma. For the preliminary futility analysis, they enrolled seventeen patients with progressive or recurrent glioblastoma and a history of treatment with surgery, radiation, and temozolomide. The median age of 


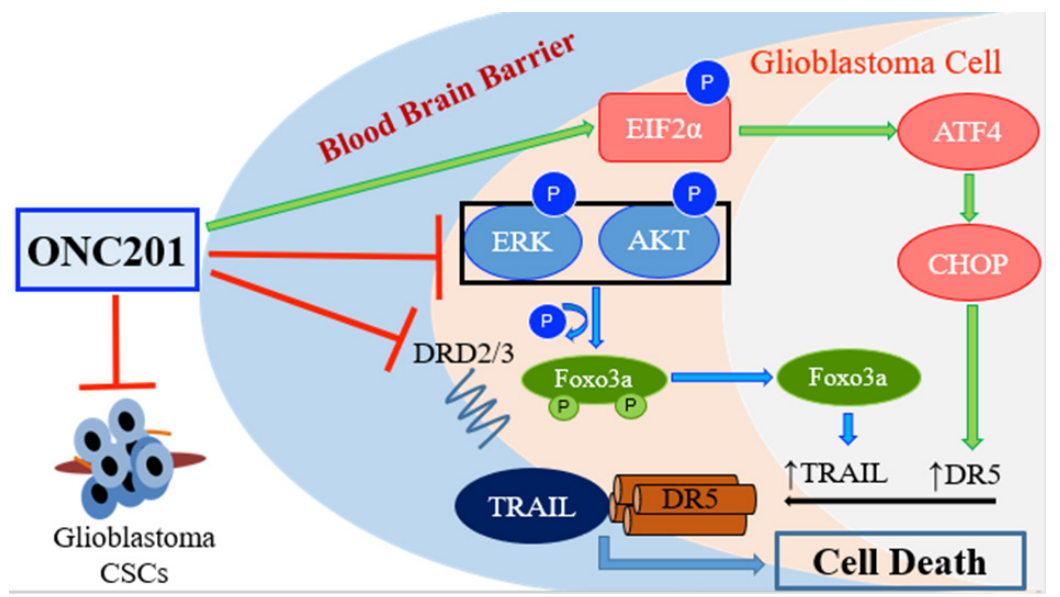

Figure 1 Mechanism of action of ONC201 in glioblastoma. Small molecule ONC201 crosses the blood brain barrier and exerts it effects against glioblastoma bulk tumor cells and cancer stem cell-like (CSC) cells. ONC201 antagonizes dopamine receptors DRD2 and DRD3. The link between dopamine receptor antagonism by ONC201 and the downstream cell death signaling pathways perturbed by ONC201 is continuing to be unraveled. ONC201 dually inhibits phosphorylation of Akt and ERK, leading to the dephosphorylation of transcription factor FOXO3A. Dephosphorylated FOXO3A translocates into the nucleus where it activates transcription of its target genes, including pro-apoptotic death receptor ligand TNF-related apoptosis inducing ligand (TRAIL). ONC201 also activates the integrated stress response (ISR) through stimulation of EIF2 $\alpha$ kinases. Phosphorylation of EIF2 $\alpha$ leads to induction of transcription factors ATF4 and CHOP, which then increase DR5 expression. The combined upregulation of the ligand TRAIL and its surface receptor DR5 by ONC201 ultimately triggers cancer cell death.

the patients was 57 years old and the median time from initial diagnosis to enrollment was 17 months. ONC201 was given once every 3 weeks (21-day cycle) as an oral capsule and was continued until tumor progression. The progression free survival at 6 months (PFS6) was $11.8 \%$, and two patients remained on therapy for greater than 11 months. The median OS of patients enrolled in the trial was 41.6 weeks, with the OS at 6 months (OS6) being 71\%.

A 22-year-old female patient with a recurrent secondary glioblastoma achieved a partial response as measured by the RANO criteria (27). This patient's tumor had a histone H3.3 K27M mutation, a mutation that is present with a high frequency in pediatric glioblastomas. Patients with mutant H3.3 have a poorer OS than those with the wild-type form of the protein (28). The patient's response was sustained for greater than 6 months, an exciting observation given the dismal prognosis for recurrent glioblastoma. The authors include the patient's contrast-enhanced MRI scans which show a dramatic reduction in the size of two intracranial lesions from baseline following treatment with 12 cycles of ONC201. Despite one incident of grade III neutropenia and a grade II allergic reaction, the compound was tolerated well overall. This is in agreement with observations about its safety made in the first-in-human trial (15). A biomarker of D2-like dopamine receptor antagonism, prolactin, increased in the serum of the patients in the study.

The results of this study are encouraging, as ONC201 showed preliminary signs of efficacy in patients with an aggressive, treatment-resistant subset of glioblastomas. ONC201 deserves further investigation in a larger cohort of patients with this deadly disease. The ONC201-glioblastoma clinical trial is continuing with an expansion cohort at Massachusetts General Hospital (MGH). There are other open trials of ONC201 in solid tumors (including at Fox Chase Cancer Center and the Cancer Institute of New Jersey) that allow enrollment of patients with glioblastoma. It will be interesting to follow the development of ONC201 in this type of tumor as progression free and OS data accumulate in a larger cohort of patients.

\section{Acknowledgments}

Funding: The authors acknowledge NIH grant R01 CA173453 (to WS El-Deiry) and support from an American 
Cancer Society Research Professorship (to WS El-Deiry).

\section{Footnote}

Provenance and Peer Review: This article was commissioned and reviewed by the Section Editor Ning Huang (Department of Neurosurgery, the Second Affiliated Hospital of Chongqing Medical University, Chongqing, China).

Conflicts of Interest: WS El-Deiry has ownership interest (including patents) in Oncoceutics. The other authors have no conflicts of interest to declare.

Ethical Statement: The authors are accountable for all aspects of the work in ensuring that questions related to the accuracy or integrity of any part of the work are appropriately investigated and resolved.

Open Access Statement: This is an Open Access article distributed in accordance with the Creative Commons Attribution-NonCommercial-NoDerivs 4.0 International License (CC BY-NC-ND 4.0), which permits the noncommercial replication and distribution of the article with the strict proviso that no changes or edits are made and the original work is properly cited (including links to both the formal publication through the relevant DOI and the license). See: https://creativecommons.org/licenses/by-nc-nd/4.0/.

\section{References}

1. Stupp R, Hegi ME, Mason WP, et al. Effects of radiotherapy with concomitant and adjuvant temozolomide versus radiotherapy alone on survival in glioblastoma in a randomised phase III study: 5-year analysis of the EORTC-NCIC trial. Lancet Oncol 2009;10:459-66.

2. Louis DN, Perry A, Reifenberger G, et al. The 2016 World Health Organization Classification of Tumors of the Central Nervous System: a summary. Acta Neuropathol 2016;131:803-20.

3. Dinapoli RP, Brown LD, Arusell RM, et al. Phase III comparative evaluation of PCNU and carmustine combined with radiation therapy for high-grade glioma. J Clin Oncol 1993;11:1316-21.

4. Walker MD, Alexander E, Hunt WE, et al. Evaluation of $\mathrm{BCNU}$ and/or radiotherapy in the treatment of anaplastic gliomas. J Neurosurg 1978;49:333-43.

5. Stupp R, Mason WP, van den Bent MJ, et al. Radiotherapy plus concomitant and adjuvant temozolomide for glioblastoma. N Engl J Med 2005;352:987-96.

6. Hegi ME, Diserens AC, Gorlia T, et al. MGMT Gene Silencing and Benefit from Temozolomide in Glioblastoma. N Engl J Med 2005;352:997-1003.

7. Ochs K, Kaina B. Apoptosis Induced by DNA Damage O6-Methylguanine Is Bcl-2 and Caspase-9/3 Regulated and Fas/Caspase-8 Independent. Cancer Res 2000;60:5815-24.

8. Patel AP, Tirosh I, Trombetta JJ, et al. Single-cell RNAseq highlights intratumoral heterogeneity in primary glioblastoma. Science 2014;344:1396-401.

9. Chen J, Li Y, Yu TS, et al. A restricted cell population propagates glioblastoma growth after chemotherapy. Nature 2012;488:522-26.

10. Gilbert MR, Dignam JJ, Armstrong TS, et al. A randomized trial of bevacizumab for newly diagnosed glioblastoma. N Engl J Med 2014;370:699-708.

11. Chinot OL, Wick W, Mason W, et al. Bevacizumab plus radiotherapy-temozolomide for newly diagnosed glioblastoma. N Engl J Med 2014;370:709-22.

12. Wagner J, Kline CL, Pottorf RS, et al. The angular structure of ONC201, a TRAIL pathway-inducing compound, determines its potent anti-cancer activity. Oncotarget 2014;5:12728.

13. Wagner J, Kline CL, El-Deiry W. Resistance to TRAIL Pathway-Targeted Therapeutics in Cancer. In: TRAIL, Fas Ligand, TNF and TLR3 in Cancer. New York, New York: Springer, 2017:1-25.

14. Allen JE, Kline CLB, Prabhu VV, et al. Discovery and clinical introduction of first-in-class imipridone ONC201. Oncotarget 2016;7:74380.

15. Stein MN, Bertino JR, Kaufman HL, et al. First-in-human Clinical Trial of Oral ONC201 in Patients with Refractory Solid Tumors. Clin Cancer Res 2017;23:4163-9.

16. Allen JE, Krigsfeld G, Mayes PA, et al. Dual inactivation of Akt and ERK by TIC10 signals Foxo3a nuclear translocation, TRAIL gene induction, and potent antitumor effects. Sci Transl Med 2013;5:171ra17.

17. Allen JE, Krigsfeld G, Patel L, et al. Identification of TRAIL-inducing compounds highlights small molecule ONC201/TIC10 as a unique anti-cancer agent that activates the TRAIL pathway. Mol Cancer 2015;14:99.

18. Ralff MD, Kline CL, Kucukkase OC, et al. ONC201 Demonstrates Antitumor Effects in Both Triple-Negative and Non-Triple-Negative Breast Cancers through TRAILDependent and TRAIL-Independent Mechanisms. Mol Cancer Ther 2017;16:1290-8. 
19. Talekar MK, Allen JE, Dicker DT, et al. ONC201 induces cell death in pediatric non-Hodgkin's lymphoma cells. Cell Cycle 2015;14:2422-8.

20. Zhang Q, Wang H, Ran L, et al. The preclinical evaluation of TIC10/ONC201 as an anti-pancreatic cancer agent. Biochem Biophys Res Commun 2016;476:260-6.

21. Ishizawa J, Kojima K, Chachad D, et al. ATF4 induction through an atypical integrated stress response to ONC201 triggers p53-independent apoptosis in hematological malignancies. Sci Signal 2016;9:ra17.

22. Kline CL, Van den Heuvel AP, Allen JE, et al. ONC201 kills solid tumor cells by triggering an integrated stress response dependent on ATF4 activation by specific eIF2 $\alpha$ kinases. Sci Signal 2016;9:ra18.

23. Prabhu VV, Allen JE, Dicker DT, et al. Small-molecule ONC201/TIC10 targets chemotherapy-resistant colorectal cancer stem-like cells in an Akt/Foxo3a/ TRAIL-dependent manner. Cancer Res 2015;75:1423-32.

24. Prabhu VV, Lulla AR, Madhukar NS, et al. Cancer stem cell-related gene expression as a potential biomarker of

Cite this article as: Ralff MD, Lulla AR, Wagner J, El-Deiry WS. ONC201: a new treatment option being tested clinically for recurrent glioblastoma. Transl Cancer Res 2017;6(Suppl 7):S1239-S1243. doi: 10.21037/tcr.2017.10.03 response for first-in-class imipridone ONC201 in solid tumors. PloS One 2017;12:e0180541.

25. Madhukar NS, Khade P, Huang L, et al. A New Big-Data Paradigm For Target Identification And Drug Discovery. bioRxiv 2017. Available online: https://www.biorxiv.org/ content/early/2017/05/07/134973

26. Arrillaga-Romany I, Chi AS, Allen JE, et al. A phase 2 study of the first imipridone ONC201, a selective DRD2 antagonist for oncology, administered every three weeks in recurrent glioblastoma. Oncotarget 2017. doi. org/10.18632/oncotarget.17837

27. Wen PY, Macdonald DR, Reardon DA, et al. Updated response assessment criteria for high-grade gliomas: response assessment in neuro-oncology working group. J Clin Oncol 2010;28:1963-72.

28. Khuong-Quang DA, Buczkowicz P, Rakopoulos P, et al. K27M mutation in histone H3.3 defines clinically and biologically distinct subgroups of pediatric diffuse intrinsic pontine gliomas. Acta Neuropathol 2012;124:439-47. 